Mots. Les langages du politique

$83 \mid 2007$

Dire la démocratie aujourd'hui

\title{
Le paradigme localiste au secours de l'action publique démocratique
}

Philippe Genestier, Samira Ouardi et Juliette Rennes

\section{(2) OpenEdition \\ 1 Journals}

Édition électronique

URL : https://journals.openedition.org/mots/907

DOI : $10.4000 /$ mots. 907

ISSN : 1960-6001

Éditeur

ENS Éditions

Édition imprimée

Date de publication : 1 mars 2007

Pagination : 69-80

ISBN : 978-2-84788-111-0

ISSN : 0243-6450

Référence électronique

Philippe Genestier, Samira Ouardi et Juliette Rennes, « Le paradigme localiste au secours de l'action publique démocratique », Mots. Les langages du politique [En ligne], 83 | 2007, mis en ligne le 01 mars 2009, consulté le 23 avril 2022. URL : http://journals.openedition.org/mots/907 ; DOI : https://doi.org/ $10.4000 /$ mots. 907

\section{(C) ENS Éditions}




\section{Le paradigme localiste au secours de l'action publique démocratique}

On note, depuis le début des années quatre-vingt-dix, dans les discours des politiques sociales françaises, à l'échelle locale autant qu'à l'échelle nationale, un usage intensif des syntagmes cité, citoyenneté, civilité, urbanité et espace public. Cet usage récurrent s'inscrit dans un réseau discursif qui tend à indexer le politique à l'urbain. Tout se passe comme si la ville faisait figure à la fois de cadre d'analyse des problèmes politiques et sociaux et de cadre de résolution de ces derniers. On sait par ailleurs que les syntagmes cité, citoyenneté et espace public renvoient à deux modèles de démocratie traditionnellement distincts, voire opposés. Il est d'usage, en effet, en sciences politiques et sociales, d'établir une forte distinction entre, d'une part, une acception étatique et républicaine (universaliste) de la démocratie, et d'autre part, une acception civile d'inspiration libérale, au sens philosophique du terme. La première acception se réfère à la nation, au sein d'un imaginaire holiste et souverainiste, la seconde au local constitué en échelle pertinente de définition de la société civile. Or, il semblerait que, malgré cette différence de sens, ces deux acceptions soient conjointement présentes, voire confondues dans les discours contemporains qui présentent, justifient, théorisent et commentent les politiques sociales. Si l'engouement pour la ville et le local ne surprend guère de la part des acteurs locaux qui, par nature, ont à produire des déclarations calibrées sur les questions dont ils sont en charge, comment interpréter le fait qu'il investisse les discours des acteurs gouvernementaux de manière si massive? Pourquoi la «politique de la ville », longtemps considérée comme un type périphérique et expérimental d'action publique, contribue-t-elle, depuis le début des années quatre-vingt-dix, à la reformulation d'une bonne part des questions sociopolitiques et, par là même,à la reformulation de la conception de la démocratie ${ }^{1}$ ? On constate qu'un cadre

1. Sur ce point, voir par exemple Éric Macé (1999). La gestion urbaine de la question sociale s'institutionnalise au cours des années quatre-vingt avec la réponse politique apportée aux troubles qui se sont déroulés dans certaines banlieues, de ceux de Vénissieux, en 1981, jusqu'à ceux de Vaulx-en-Velin en 1990. Cette institutionnalisation se concrétise la même année par la création du ministère de la Ville. 
de pensée et de discours organisé autour de termes référés au local et à la ville, ce que l'on pourrait appeler un cadre urbain de la pensée politique, est porté par une large part des élus de tous bords, tant locaux que nationaux. Comment expliquer que ces discours excèdent la sphère institutionnelle au point qu'on les retrouve aussi bien dans de multiples proclamations militantes et associatives que sous la plume d'universitaires et de chercheurs en sciences sociales? Quelles contraintes sociopolitiques et économiques motivent cet infléchissement discursif et quelle logique interne au champ du pouvoir le structure? Quel imaginaire de la démocratie véhicule-t-il ?

\section{Corpus et outil d'analyse}

Nous avons confronté ces interrogations à un corpus de discours étatiques (ministériels et d'expertise) émis sous le gouvernement de Lionel Jospin (19972002) et rassemblés au sein d'une étude plus large sur les cadres cognitifs et discursifs de l'action publique contemporaine ${ }^{2}$. Ce corpus se compose de 275 prises de parole ministérielles, provenant de douze locuteurs ${ }^{3}$ et de trois rapports ministériels relatifs aux questions socio-urbaines ${ }^{4}$. Nous avons soumis ce corpus à une analyse lexicale afin de caractériser les ambigüités sémantiques et référentielles dans l'emploi des syntagmes cité, civilité, urbanité, citoyenneté et espace public. L'analyse de ces usages, qui visait à mettre au jour les représentations du monde social et de l'action politique mobilisées dans le corpus, s'est partiellement appuyée sur certaines fonctionnalités du logiciel de traitement automatique du discours Weblex ${ }^{5}$ : des recherches contextuelles ou concordances à partir de mots-pivots, et un repérage des segments répétés, ou

2. 2004: "Cité », " citoyenneté » et "espace public ». Les opérations cognitives et discursives de l'action publique contemporaine. Rapport financé par l'Action concertée incitative-ville (ACIV) du ministère de la Recherche, réalisé dans le cadre du laboratoire Théorie des mutations urbaines (CNRS).

3. Il s'agit des ministres suivants: Claude Allègre (Éducation), Martine Aubry (Solidarité), Claude Bartolone (délégué à la Ville), Louis Besson (délégué au Logement), Marie-George Buffet (Sports), Jean-Pierre Chevènement (Intérieur), Elisabeth Guigou (Justice), Lionel Jospin (Premier ministre), Marie-Noëlle Lienemann (déléguée au Logement), Dominique Strauss-Kahn (Économie), Dominique Voynet (Environnement). Le corpus, formé de discours publics et de prises de parole dans les médias, a été en partie constitué dans le cadre d'une étude précédente sur $L a$ communication gouvernementale de la gauche plurielle (Simone Bonnafous, Caroline OllivierYaniv, Pierre Fiala, Juliette Rennes). Rapport commandé et financé par le Service d'information du gouvernement (SIG), réalisé dans le cadre du CEDITEC, Université Paris 12, et du laboratoire Lexicométrie et textes politiques (ENS-LSH, CNRS).

4. Territoires, emploi et développement durable : rapport au Premier ministre de Guy Hascoet, député du Nord, octobre 1997; Le territoire de la cité au service de l'emploi : rapport au Premier ministre de Chantal Robin-Rodrigo, députée des Hautes-Pyrénées et Pierre Bourguignon, député de Seine-Maritime, juin 1999. Conclusions du groupe de travail interministériel sur les emplois dits de médiation sociale, présentées parYvon Robert (président du groupe de travail) le 14 juin 2001.

5. Créé et mis en ligne par Serge Heiden, ENS-LSH, CNRS (Icar). 
expressions figées, statistiquement significatives, qui permettent d'identifier des phraséologies et des phénomènes de sloganisation $^{6}$ (ex. : « service public citoyen », « exercice de la citoyenneté », « sur le terrain », etc.).

La première partie de ce travail s'est concentrée sur l'étude des glissements d'acception de ces mots à travers ces différents discours. Cette approche lexicale a servi de point d'appui à une analyse des cadres cognitifs et des structures de raisonnement implicites communs à tous ces textes et qui semblent transcender les usages, pourtant théoriquement opposés, de ces notions. Il apparait en effet que la référence à la ville et au local se déploie selon des procédés réguliers qui dessinent, à terme, une véritable structure argumentative dont la cohérence interne et la prégnance suggèrent qu'elle est l'expression d'un paradigme plus large.

\section{Quand l'urbain reconfigure le politique}

Les recherches contextuelles à partir des mots étudiés font apparaitre une tendance au syncrétisme sémantique entre les questions urbaines et sociales. Cette fusion/confusion se construit à travers l'usage d'un certain nombre de figures rhétoriques qui s'appuient sur les potentiels polysémiques du lexique. La syllepse, soit l'usage simultané des sens propre et figuré d'un mot, constitue un trope typique de ces discours. Dans notre corpus, un exemple est constitué par l'emploi du syntagme espace public qui fond et confond l'espace physique urbain et la sphère publique délibérative ${ }^{7}$, comme lorsque Claude Bartolone proclame: «Du renouveau de la citoyenneté, d'une nouvelle manière de faire de la politique au niveau local, émergeront alors de nouveaux espaces publics, de nouveaux lieux d'échange, une nouvelle forme d'intégration " 29 septembre 1999). Ce même procédé se retrouve avec le vocable proximité, dont on motive simultanément le sens géographique concret et le sens social abstrait, la proximité spatiale étant assimilée à une absence de distance sociale. Ce présupposé s'est par exemple institutionnalisé dans les désignations «police de proximité », "services publics de proximité », "sécurité de proximité ». Les mots urbanité et civilité sont quant à eux utilisés dans leur polysémie qui renvoie à la fois au statut concret d'habitant de la ville et à une éthique plus abstraite de la politesse. Louis Besson use de façon récurrente de cette syllepse pour suggérer que la condition citadine constitue en elle-même une pédagogie des bonnes mœurs: «Poser la question de nos villes sous l'angle de l'urbanité [...], c'est parler de la qualité de la vie en ville et de la manière de vivre ensemble ». Quant

6. Selon l'expression de Maurice Tournier, 1997.

7. Le titre du fameux livre de Jürgen Habermas, Strukturwandel der Öffentlichkeit (1962), a été traduit L'espace public en français, alors que sa traduction anglaise, plus fidèle, est The Structural Transformation of the Public Sphere: An Inquiry into a Category of Bourgeois Society. 
à Claude Bartolone, c'est plutôt à partir du terme « cité » qu'il tient ce même discours de la citadinité civilisée et civilisatrice : il valorise «la parole des habitants, parole sans laquelle il n'y a pas de cité, sans laquelle il n'y a pas de ville » (27 mai 2000).

Qu'ils concernent la partie pour le tout, le contenant pour le contenu, l'effet pour la cause ou le lieu pour l'objet, les procédés métonymiques entretiennent eux aussi, de façon récurrente, cette confusion du social et du spatial. Dans des formules qui assument cette rhétorique, on affirme par exemple que «la cage d'escalier représente en quelque sorte l'espace public » (Marie-Noëlle Lienemann, 8 juin 2001), ou encore que « les quartiers sont devenus le "concentré" de tous nos maux» (Martine Aubry, 13 janvier 1998). Dans le même ordre d'idées, Louis Besson évoque la ville comme «caisse de résonance de toutes les inégalités sociales » (10 décembre 1999). Le lieu vaut en tant qu’il désigne la population qu'il contient ; à l'inverse, la population contenue n'existe qu'en tant que groupe spécifique justiciable d'une évaluation propre et bénéficiaire d'une action ciblée: Martine Aubry évoque ainsi «l'aide aux quartiers qui ont aujourd'hui des difficultés » (4 juin 1998). Le partage d'une même localisation constituerait un facteur unificateur et identificateur: c'est le présupposé de l'expression « jeunes des quartiers ».

L'étymologisme, soit l'usage d'une racine étymologique commune comme argument pour établir une parenté sémantique et/ou référentielle entre divers termes, constitue une autre figure qui participe dans notre corpus du cadrage urbain des questions sociales. Ainsi, la référence explicite à l'origine latine civis des mots comme cité, citoyenneté, civilité, civilisation vise bien souvent à gommer la distinction entre l'urbs et la civitas ${ }^{8}$ : «être citoyen, c'est aussi être acteur dans la cité, s’impliquer dans la vie collective », déclare ainsi Claude Bartolone (20 septembre 1999). Quant à Louis Besson, il fait valoir encore plus explicitement qu' «aménager la cité sans l'implication des citoyens ne peut en effet que conduire à une impasse. Les habitants veulent passer du statut de CITADIN usager au statut légitime de citadin citoyen » (8 mars 2000 [en majuscules dans le texte]) ${ }^{9}$. Cet étymologisme est souvent implicite, comme lorsque

8. Dans l'Antiquité romaine, comme en Grèce, on distinguait deux réalités à l'intérieur de la notion de ville en son sens actuel. Les Anciens utilisaient le terme civis pour définir une communauté politique et le terme urbs pour indiquer l'espace physique de la ville, en tant que domaine bâti concentrant une population.

9. Cet usage particulier de l'étymologisme n'est pas propre à la sphère gouvernementale : il tend à investir l'ensemble du discours contemporain français sur la citoyenneté. On le retrouve en particulier dans les interventions publiques d'intellectuels au sujet de la crise sociale. Par exemple, François Barré, ancien directeur de l'architecture et du patrimoine au ministère de la Culture : "L'espace public est le berceau de la cité. L'agora et le forum ont inventé le politique, lieu et procédure de l'échange et du partage, entre-deux où naissent l'urbanité et la citoyenneté. Cet espace-là, symbole du projet collectif, nous ne savons plus le constituer.[...] II nous faut donc réapprendre à parler (de) la ville et refonder le politique. Une ville s'énonce et fait récit. » Manière de voir, mai-juin 2001, p. 46. 
l'on se réfère sans plus de précision à la dimension civilisatrice dont la ville serait historiquement porteuse : " La ville et la civilisation urbaine [...] sont notre destin commun. Car depuis ses origines, la ville a été le lien de l'échange et de la rencontre des autres» (Louis Besson, 8 mars 2000). Ce type de rhétorique est évidemment rendu possible par la vulgate relative à la naissance urbaine de la démocratie antique : l'urbain serait, par vocation, le lieu de la démocratie directe, soit l'archétype d'une démocratie dont l'actualisation sous la forme représentative ne serait qu'un pis-aller ${ }^{10}$.

La paronomase consiste à jouer sur des homophonies approximatives qu'en général l'étymologie ne justifie pas (spatial et social, lien et lieu...), mais qui suggèrent un rapport structurel ou une relation d'inférence entre des domaines en réalité hétérogènes. Par exemple, sous la plume de C. Bartolone : «Ce sont les lieux qui comptent: maisons de quartier, de la justice et du droit, centres culturels, mais ce sont aussi les liens, les réseaux de solidarité, les réseaux de sociabilité urbaine, les espaces symboliques» (31 mars 1999). Et sous celle de Jean-Pierre Chevènement: «La France s'est cassée. Socialement et spatialement. [...] La ségrégation spatiale redouble ainsi la ségrégation sociale [...]. Il faut donc se donner tous les moyens de lutter sur le long terme contre l'apartheid social. Et, puisqu'il revêt de plus en plus la forme d'un apartheid spatial, il faut repenser notre organisation urbaine. Relever l'idéal de la citoyenneté " (26 mai 1998).

Cette citation est archétypale du discours d'intrication de l'urbain et du social. Une telle intrication s'enracine certes dans des transformations sociologiques : elle peut être expliquée par les politiques de peuplement ségrégatives des grands ensembles périphériques, lesquelles ont eu pour conséquence de créer des espaces où se concentrent les problèmes sociaux propres aux économies post-fordistes et néolibérales ${ }^{11}$. Mais elle traduit également une transformation des cadres interprétatifs du monde social et des paradigmes de l'action publique qui concerne tout autant l'échelle d'analyse et de traitement des problèmes politiques que l'ethos des acteurs gouvernementaux et publics.

10. Sur les usages contemporains de l'imaginaire de la démocratie antique, voir notamment Finley, 1976.

11. Parmi beaucoup d'autres analyses, citons Éric Maurin (2004) qui analyse la rupture, depuis une génération, par rapport au modèle de production et de consommation de masse issu des Trente glorieuses ainsi que ses conséquences sur le mode de vie des anciennes couches ouvrières et sur leur répartition dans les agglomérations urbaines. Ainsi, les grands ensembles HLM, d'abord hauts lieux de la « moyennisation » sociale, devinrent les réceptacles des catégories fragilisées. 


\section{Le terrain, le local, le « bottom up » : les nouvelles échelles de l'action publique}

Le «quotidien », le «terrain » et l'acteur social localisé tendent à devenir à la fois les destinataires principaux et les détenteurs des critères de validité de l'action publique : celle-ci se conjugue désormais à une micro-échelle spatiotemporelle. Ce paradigme se fige notamment dans des segments répétés comme de proximité, sur le terrain, au plus près de, au quotidien, du terrain, proximité avec le terrain, réalité du terrain, au contact de, au quotidien... Pour justifier leurs décisions, les responsables politiques se targuent par exemple de revenir « désormais à des approches concrètes, mesurables, avec une volonté : la proximité avec le terrain » (Dominique Voynet, 17 septembre 1997), « de privilégier une approche locale, au plus près du terrain » (Claude Bartolone, 20 septembre 1999), ou prônent une «stratégie urbaine » qui « doit se concrétiser au plus près du terrain » (Louis Besson, 10 novembre 1999).

Ce déplacement d'échelle s'accompagne d'une critique des politiques planificatrices et centralisées et témoigne d'une croyance inédite dans l'action progressive, au coup par coup, itérative, partenariale, négociée et quotidienne ${ }^{12}$. L'instaurationnisme ${ }^{13}$ cède la place à une forme de continuisme et d'empirisme, comme le proclame par exemple Claude Bartolone :

C'est en travaillant à améliorer les petites choses simples de la vie quotidienne, tout ce qui grippe, qui coince, qui empêche, tous ces accrocs du temps qui nous font vivre la ville sur un tempo disharmonieux, voire insupportable, qu'on peut tout simplement donner envie aux habitants de s'impliquer davantage dans la vie de leur cité. (18 juin 2001)

Les citations de ce type, qui témoignent d'une conception modérée et modeste de l'action publique, sont légion. Ainsi Marie-Noëlle Lienemann assure-t-elle :

L'essentiel, c'est que sur le terrain des petites choses régulièrement améliorées fassent réapparaître l'idée de progrès dont je vous disais qu'il était parfois en crise dans l'esprit de nos concitoyens qui vivent dans les quartiers. (22 juin 2001)

L'urbanité républicaine, c'est aussi le citoyen au quotidien, car je me méfie des «demain on rase gratis», des «j'ai un grand projet pour l'avenir» qui d'ailleurs, quand on y regarde de plus près, ne sont jamais totalement achevés et pendant ce temps-là le quotidien se détériore. (4 octobre 2001)

12. Sur ce point, voir notamment, Bourdin, 2003 et Gaudin, 1999.

13. On désigne par ce terme l'idée - et l'idéal - selon lesquels la puissance publique instaure le système social. Voir le chapitre intitulé « l’État instituteur du social » dans Rosanvallon, 1990. 
Dans le même ordre d'idées, Jean-Pierre Chevènement, pourtant chantre depuis trente ans d'une conception souverainiste de l'action publique, affirme : « Je ne crois pas aux règlements, aux lois pour changer la vie, je crois que le changement se fait sur le terrain [et qu'] il faut faire vivre au quotidien le projet d'une société citoyenne » (26 mai 1998).

Le versant positif de ce scepticisme affiché envers les politiques publiques centralisées, autoritaires et bureaucratiques est l'éloge de la fondation locale de la décision et d'un fonctionnement démocratique allant du bas vers le haut, ce que la science politique a par ailleurs décrit comme le basculement d'un mode de gouvernement «top-down » vers une gouvernance «bottom-up». «Ce n'est pas du tout la peine d'aller remonter au niveau national ce qui peut se réaliser sur le terrain », affirme Marie-Noëlle Lienemann (11 mai 2001). L'usage récurrent de syntagmes verbaux comme remonter de ou partir de est caractéristique de ce discours. Jean-Pierre Chevènement conseille de procéder à « la collecte des symptômes multiples remontant du terrain » (19 janvier 1998 [nous soulignons]), et Martine Aubry de «partir des villes telles qu'elles sont » (13 janvier 1998 [nous soulignons]). Dans leur rapport officiel sur Le territoire de la cité au service de l'emploi, Chantal Robin-Rodrigo et Pierre Bourguignon estiment que « la politique de la ville [...] doit aboutir à une définition partagée d'objectifs, partant d'une analyse des problèmes locaux et des opportunités de terrain » ( $1^{\mathrm{er}}$ juin 1999 [nous soulignons]).

Cette notion de « terrain » est révélatrice de la valorisation de l'échelle locale comme étalon de la démocratie. Une échelle dont la figure positive est la ville, par opposition à la banlieue ${ }^{14}$. Les discours mettent régulièrement en scène la pluralité des acteurs locaux, dont l'expérience fait office de précieuse expertise, comme moteurs de changements socio-politiques. Marie-Noëlle Lienemann assure avoir «beaucoup appris du commissaire de police de [sa] commune » (8 juin 2001), tandis que Claude Bartolone déclare que « la politique de la ville a besoin des associations, de l'ensemble des acteurs de terrain, des administrations » et qu'elle "s'appuie souvent sur les réseaux associatifs présents» (20 septembre 1999). Plus loin, il affirme que «c'est localement, au plus proche des habitants que la collectivité se construit. Ce mouvement ne peut être défini par l'État central, il doit être inventé par les acteurs locaux » (même date). Cette nouvelle échelle fonctionne de pair avec une modification de l'ethos de l'acteur politique. En effet, le terrain fonctionne comme élément de légitimation des politiques publiques autant que du rôle institutionnel de leurs responsables.

14. Alors que cité, renvoyant à l'échelle de l'agglomération, peut désigner un idéal par référence à son étymologie latine, il s'avère que, lorsqu'il est employé à l'échelle du quartier (souvent au pluriel), le mot évoque les grands ensembles HLM de banlieues. Les cités font alors figure de spatialité négative caractérisée par une carence de « civilité » et d' " urbanité ». 


\section{Entre modestie et volontarisme, un nouvel ethos politique}

Ministres et élus rivalisent pour répondre à l'idéal du technicien réaliste pouvant afficher son vécu et ses expériences concrètes. Lionel Jospin affirme: «Moi, je discute sur le terrain [...]. Je veux être au contact du terrain » (21 janvier 1998). Dans un exposé sur sa politique en matière de constructions HLM, Marie-Noëlle Lienemann argumente: «Parce qu'observatrice de terrain, je sais qu'il est des lieux où les élus locaux souhaitent des opérations de cette nature » (11 mai 2001), et Guy Hascoet déclare : "Le développement local fait surtout appel à une culture empirique qui s'apprend en grande partie par le travail de terrain » (15 octobre 1997). S'être rendu "sur le terrain» constitue un argument d'autorité pour faire valoir la pertinence de ses cadres d'analyse politique. Dans son plaidoyer à l'Assemblée nationale pour la loi SRU (Solidarité renouvellement urbain), Louis Besson explique : «Ce texte résulte d'une écoute attentive des élus, des représentants de la société civile, représentatifs du monde urbain, des professionnels et des experts, ceux d'en haut et ceux du terrain, du quotidien » (8 mars 2000). Remarquons que ces déclarations ministérielles, prononcées par des membres d'un gouvernement de gauche, auraient pu être émises par un élu local d'opposition : «Je ne crois qu'au terrain et au dialogue [...]. Le maire sur le terrain règle les problèmes de ses administrés. Je crois bien connaître mes électeurs, ils m'écrivent, ils me parlent. Pendant ces derniers mois [...], sans battage ni trompette, j'ai beaucoup travaillé » (Jean Tiberi, 23 novembre 1999) ${ }^{15}$.

Cette valorisation du terrain et de l'action localisée et modeste fonctionne de pair avec l'imaginaire d'une société frappée de dysfonctionnements et de malaises locaux, lesquels requièrent une intervention réparatrice des pouvoirs publics. La manifestation lexicale la plus remarquable de cet imaginaire est l'emploi abondant du vocabulaire formé à partir du préfixe dé-, celui de la dégradation et du désordre. Au diagnostic formulé en dé- répond quasi automatiquement une thérapeutique en re-, qui définit les visées de l'action publique. Si l'usage politique de ces préfixes est historiquement connoté à droite, voire à l'extrême droite, le dé- et le re- des politiques urbaines et sociales ne s'appliquent pas aux mêmes champs lexicaux que ceux de la droite réactionnaire traditionnelle. Le vocabulaire organiciste (décrépitude, dégénérescence, décadence...) et celui de la restauration et du redressement de type nationaliste, autoritaire et moral (tel qu'il s'est exprimé durant la «Révolution nationale»

15. Voir aussi sur ce point les stratégies de légitimation de l'action gouvernementale par le Premier ministre de droite, Jean-Pierre Raffarin, analysées par Christian Le Bart, 2005 et par Sylvianne Rémi-Giraud, 2005. 
vichyste et tel qu'il se manifeste encore dans les discours du Front national $\left.{ }^{16}\right)$ ne sont absolument pas mobilisés dans ces discours.

Dans notre corpus, le vocabulaire en dé- est associé à deux référents, souvent mêlés : un premier, de type concret et physique, renvoyant au délabrement de l'habitat, des logements, des infrastructures publiques; un second, de type immatériel, désignant les comportements politiques et les relations sociales. Ces usages témoignent ainsi de la logique générale d'indexation du social à l'urbain, comme lorsque Claude Bartolone entend s'attaquer aux "quartiers anciens marqués par une profonde dégradation urbaine et sociale» (17 mai 2001), assigne au grand projet de ville l'objectif de trouver des solutions « au cadre de vie dégradé » des habitants des quartiers populaires (17 mai 2001), diagnostique une « citoyenneté [...] en désarroi » (18 mai 1999) et un « dérèglement urbain » (1 $1^{\text {er }}$ octobre 2000). Cette ambivalence du vocabulaire préfixé par -dé traverse également les déclarations de Louis Besson qui évoque à plusieurs reprises « la spirale de la dégradation » touchant « certains territoires urbains » (3o novembre 1999), un "dépérissement de certains quartiers existants» (14 mars 2000), des "désordres territoriaux et sociaux» (6 octobre 1999); ou de Chantal Rodrigo et Pierre Bourguignon qui dénoncent «la spirale de la paupérisation et de la dévalorisation urbaine » dans les «quartiers » ( $1^{\mathrm{er}}$ juin 1999).

La thérapeutique en re-se décline selon plusieurs termes. II peut s'agir de réparer, restaurer, réinstaurer l'acquis : "La médiation sociale est définie comme un processus de création et de réparation du lien social et de règlement des conflits de la vie quotidienne », est-il par exemple expliqué dans le rapport d'Yvon Robert (14 juin 2001). Mais aussi de le reconstruire et le reconquérir - Claude Bartolone insiste à plusieurs reprises sur la nécessité de « reconquérir l'urbanité » (18 mai 1999) ou "l'harmonie urbaine» ( $1^{\text {er }}$ octobre 2000) - ou encore de rassembler pour créer du lien social, selon un paradigme communicationnel constitutif des discours politiques contemporain $\mathrm{s}^{17}$ : Louis Besson appelle de ses vœux la réactivation du rôle « de brassage, de mélange, de rassemblement qui est historiquement [celui de la ville] » (7 juillet 1999), et Claude Allègre préconise «de se rassembler autour de valeurs communes pour effectuer l'intégration » (6 février 1998). On note à cet égard que la thérapeutique en re- est souvent accompagnée d'injonctions à communiquer et communier. Celles-ci se traduisent dans le corpus par la forte présence, aux côtés du re-, d'un vocabulaire formé sur le préfixe co- qui sert à désigner tant les finalités de l'action publique - on évoquera alors davantage la cohésion, la communauté, la collectivité, la convergence - que ses modalités de réalisation - passant par la concertation, la consultation, la co-élaboration: «Nous avons besoin de

16. Voir sur ce point Tournier, 1997, «-de et -re dans le même bateau ».

17. Breton, 1991 ; Neveu, 1997. 
concertation, nous avons besoin de nous entendre, nous avons besoin de nous comprendre. C'est vrai à tous les échelons de la société, ça doit être bien sûr vrai dans l'habitat social », dit par exemple Marie-Noëlle Lienemann (22 juin 2001). Ces représentations investissent également des dispositifs législatifs (sur la démocratie participative par exemple) ou ministériels (on pense à la création ultérieure du ministère de la Cohésion sociale ${ }^{18}$ ).

Enfin, on remarque que le préfixe re- est volontiers associé au sème de la nouveauté : renouveau, renouveler, renouvellement (comme dans les opérations d'urbanisme officiellement baptisées ORU, soit « opération de renouvellement urbain »). Dans le même ordre d’idées, ce préfixe est également uni aux sèmes de la vitalité, de la naissance, de la croissance : réactivation, revitalisation, renaissance, redéveloppement. Ainsi Claude Bartolone appelle de ses vœux une «véritable renaissance urbaine » et estime que «la renaissance des villes passera par un renouveau de pratiques démocratiques» (4 avril 2000). Ce type de politique publique préconise que l'on parte du connu, du déjà là. Il s'agit moins de proposer, d'établir, d'instaurer que de repenser, redéfinir, réorienter. Claude Bartolone affirme qu'il faut « repenser nos villes dans leur globalité, pour agir sur les causes même de l'exclusion urbaine et sociale» (8 mars 2000). Le renouvellement n'est pas la nouveauté : il se calibre autant sur le futur que sur un retour en arrière. La rhétorique du renouvellement permet de concilier continuisme et idée d'action forte, sans aller jusqu'à la rupture.

Ainsi, bien qu'apparemment peu ambitieux dans ses propositions d'action publique et dans le pouvoir qu'il s'attribue en matière de changement, le personnel politique est encore travaillé par un idéal instaurationniste. Dit autrement, l'ethos de l'acteur politique est paradoxal : il allie modestie et volontarisme, visées prométhéennes et projet situé. L'action politique locale, itérative, quotidienne, est qualifiée par des adjectifs hyperboliques et accompagnée de commentaires qui réactivent l'imaginaire instaurationniste traditionnel. Claude Bartolone affirme par exemple que «l'ambition est forte : redonner aux quartiers les plus en difficulté une place nouvelle au sein de l'agglomération » (8 mars 2000) et, ailleurs, évoque «l'ambition de transformer en profondeur ces quartiers populaires particulièrement dégradés, devenus des symboles de l'exclusion dans notre société urbaine » (17 mai 2001 [souligné dans le texte]). L'usage récurrent du syntagme civilisation urbaine est caractéristique de cette tension entre la modestie de l'action et l'ampleur de l'ambition. Il permet de qualifier de façon grandiloquente de simples opérations de réhabilitation. Louis Besson assure, par exemple, qu'il faut mettre

18. Nouveau nom du ministère des Affaires sociales sous le gouvernement Raffarin en 2002. Ce dernier exemple montre que la cohésion peut être érigée en valeur aussi bien par la droite que par la gauche. Pour la droite, il s'agira davantage de la cohésion de la nation, pour la gauche de la solidarité sociale. 
l'humain au cœur du processus. Dans ce qui est à mes yeux un vrai et grand projet de société, construire la ville qui sera celle de la civilisation urbaine, la règle d'urbanisme doit être mise au service des politiques urbaines. Elle doit faciliter le développement de projets de ville, la revitalisation des quartiers anciens et de leur habitat, la restructuration des quartiers déqualifiés. (7 juillet 1999)

Et selon Claude Bartolone :

Le renouveau des villes que nous préparons ensemble devra d'abord donner confiance aux habitants et leur procurer un ancrage dans la civilisation urbaine en marche, pour qu'ils aient le sentiment de choisir leur ville au lieu de la subir. (4 avril 2000)

Ici, la tension entre modestie et ambition s'exprime également par une construction que l'on pourrait qualifier de déceptive : la phrase s'ouvre sur des effets d'annonce prométhéens pour se clore sur des propositions d'action très limitée, dans l'espace comme dans leurs ambitions transformatrices. Dans cet énoncé, le ministre propose en effet d'agir non sur les structures de la ville (entendue ici plus généralement comme métonymie du social) mais sur le sentiment que les individus ont de cette structure, autrement dit par des politiques de communication.

\section{Produire de la communauté pour sauvegarder l’agir politique?}

Dans l'ensemble de ces discours, les solutions présentées pour régler les problèmes publics correspondent le plus souvent à des mesures de façade: au sens propre du terme, des interventions sur le bâti, et au sens figuré, un travail de polissage des mœurs. Il est préconisé à la fois d'agir sur l'aspect physique et esthétique des grands ensembles pour pacifier les comportements, de diffuser la civilité et d'en attendre des effets d'entrainement sur les comportements sociaux.

Comment se fait-il que ces interventions localisées fassent l'objet d'une telle emphase ? On peut faire l'hypothèse que ce paradoxe est aujourd'hui discursivement acceptable, voire idéologiquement souhaitable, parce qu'il permet de préserver la croyance en la capacité de l'instance politique à orchestrer du devenir collectif. En effet, la décentralisation autant que la montée en force de la thématique de la « démocratie participative » signent la crise du monopole de la formulation légitime des problèmes publics par l'État et la perte de crédibilité de ce dernier lorsqu'il propose des solutions. Ce phénomène est renforcé par les effets de la globalisation de l'économie et le poids des directives européennes, corollaire de la crise de la nation comme cadre d'appartenance. Le local comme échelle décisionnelle et comme valeur politique est, dans ce 
contexte, convoqué comme argument de relégitimation de la décision publique. L'enjeu de cette posture est de préserver le présupposé de l’imaginaire « institutionnaliste " ou "édificationiste $~^{19}$ selon lequel les pouvoirs publics ont la mission de rassembler et unifier le corps social. Sa réaffirmation incantatoire contemporaine apparait comme une tentative de préservation d'une certaine conception du politique dont les attributs sont transférés de l'échelle nationale à l'échelle locale. Autrement dit, l'usage surabondant des figures de rhétorique qui fondent la politique de la ville, et qui nourrissent plus largement l'orientation localiste et urbaniciste de l'action publique, participerait d'un mode de pensée inédit et intermédiaire : celui où le politique revendique tous ses attributs symboliques mais, pour ce faire, ne se positionne qu'à une micro-échelle d’intervention, ce qui de facto semble démentir ses prétentions.

\section{Références}

BOURDIN Alain, 2003, La question locale, Paris, Presses universitaires de France.

BRETON Philippe, PROULX Serge, 1991, L'explosion de la communication : la naissance d'une nouvelle idéologie, Paris, La Découverte.

FINLEY Moses, 1976, Démocratie antique et démocratie moderne, Paris, Payot. FOUCAULT Michel, 2001, Dits et écrits, vol. 2., Paris, Gallimard.

-2004, Sécurité, territoire, population, Paris, Gallimard.

GAUDIN Jean-Pierre, 1999, Gouverner par contrat, Paris, Presses de Sciences Po.

LE BART Christian, 2005, «La proximité selon Raffarin », Mots. Les langages du politique,

$\mathrm{n}^{\circ} 77$, mars.

MACÉ Éric, 1999, «Les violences dites “urbaines” et la ville : du désordre public au conflit dans l'espace public », Les Annales de la recherche urbaine, $n^{\circ}$ 83-84, p. 59-64.

MAURIN Éric, 2004, Le ghetto français, Paris, Le Seuil.

NEVEU Érik, 1997, Une société de communication ? Paris, Montchrestien.

RABINOW Paul, 1995, French Modern. Norms and Forms of the Social Environment, Chicago, Londres, University of Chicago Press.

RÉMI-GIRAUD Sylvianne, 2005, «France d'en haut / France d'en bas. Raffarin tout terrain », Mots. Les langages du politique, $\mathrm{n}^{\circ} 77$, mars.

ROSANVALLON Pierre, 1990, L'État en France de 1789 à nos jours, Paris, Le Seuil. TOURNIER Maurice, 1997, Des mots en politique, Paris, Klincksieck.

19. Au sens analysé par Paul Rabinow (1995), qui s'est beaucoup inspiré de Michel Foucault et de ses analyses de «l'État pasteur » (Foucault, 2001, 2004). 